\title{
Development of LVSEM-EDS Analyzer Utilizing Superconducting X-ray Detector Toward Nanometer-scale Elemental Mapping of CFRP/Adhesive Interface
}

\author{
Go Fujii ${ }^{*}$, Masahiro Ukibe ${ }^{1}$, Shigetomo Shiki ${ }^{1}$, and Masataka Ohkubo ${ }^{1}$ \\ 1. Nanoelectronics Research Institute, National Institute of Advanced Industrial Science and Technology \\ (AIST), 1-1-1, Umezono, Tsukuba, Ibaraki, Japan. \\ *Corresponding author: go-fujii@aist.go.jp
}

Carbon fiber reinforced plastics (CFRPs) exhibit much bigger strength-to-weight ratio and better toughness than those of metals. Due to the above superior characteristics, a lot of CFRP is used in component parts of aircrafts because the lightweight structures cause many benefits like good fuel efficient and so on. Large CFRP composite structure such as wing spars and fuselage components of the aircraft should be fabricated by bonding the CFRP parts with an adhesive and bolting because the reliability of the adhesive strength between the CFRP parts hasn't been established yet due to the poor understanding of the adhesion state including chemical bonding states between CFRPs and adhesive in CFRP composite structures. An advantage of a high strength-to-weight ratio in the CFRPs can't be fully utilized so far. Therefore, to establish the reliability of the adhesive strength between the CFRP parts scientifically and to realize the CFRP composite structures constructed by using only adhesives, it is very important to evaluate amounts of each light element and their spatial distribution in the interface by performing qualitative elemental mappings of the light elements in nanometer-scale and so on, because it is necessary to evaluate diffusion of the adhesive to the inside of the CFRP and contamination of CFRP surface.

An energy-dispersive X-ray spectroscopy (EDS) analyser combined with a scanning electron microscope (SEM) is suitable to obtain spatial and quantitative information on the elemental composition of a sample non-destructively. In particular, low-acceleration-voltage SEMs (LVSEMs) theoretically allow evaluating the information of a sample surface with a nanometer lateral resolution [1]. However, it isn't suitable to use energy-dispersive X-ray detectors such as silicon drift detectors (SDDs) [2] or $\mathrm{Si}(\mathrm{Li})$ detectors in the conventional EDS analysers for obtaining X-ray spectra from samples with a low acceleration voltage condition, because the energy-resolving power of conventional energy-dispersive X-ray detectors is insufficient to clearly resolve characteristics X-ray peaks of light elements $(\mathrm{C}, \mathrm{N}, \mathrm{O}$, etc) in the CFRP and adhesive in the soft X-ray region.

In contrast, energy-dispersive X-ray detectors using arrays of superconducting-tunnel-junctions (STJs) have simultaneously exhibited excellent energy resolution of $<10 \mathrm{eV}$, relatively large detection area of $>1 \mathrm{~mm}^{2}$, and high counting rate capability of $>200 \mathrm{kcps}$ for soft X-rays less than $1 \mathrm{keV} \mathrm{[3].} \mathrm{In} \mathrm{this} \mathrm{work,}$ we have developed a LVSEM utilizing 100-pixel STJ array as an EDS system, which is abbreviate as SC-LVSEM hereafter, in order to achieve nanometer-scale mapping of light elements. In order to demonstrate X-ray detection performances of the SC-LVSEM for CFRPs at low-acceleration-voltage condition, we have performed elemental analyses of a Poly Phenylene Sulfide Resin (PPS), which is commonly used as a resin material in the CFRP.

Figure 1 shows a picture of the SC-LVSEM. The SC-LVSEM consisted of a Schottky Field Emission LVSEM and the STJ array detector. The STJ array was cooled to $0.31 \mathrm{~K}$ on a cold stage of a cryogenfree helium-3 cryostat. X-rays emitted from the sample by the electron beam were detected by the STJ 
array via the polycapillary X-ray lens [4], which is used in the system to improve the collection efficiency. The collection efficiency of the SC-LVSEM was a few mSr, which was about 1/100 times smaller than that of the SDDs.

Figure 2 shows a comparison of the X-ray spectra acquired using the STJ and SDD. The spectra were obtained in the acceleration-voltage of $5 \mathrm{kV}$. The SDD had an effective area of $25 \mathrm{~mm}^{2}$ and was equipped with an ultra-thin SiN X-ray window. The detection efficiency for C-K $\alpha$ of the STJ and the SDD were 1.7 and $32 \mathrm{cps} / \mathrm{nA} / \mathrm{bin}$, respectively, and the detection efficiency for $\mathrm{C}-K \alpha$ of the STJ was about 20 times smaller than that of the SDD. However, in the spectrum obtained by the STJ, S- $L \alpha, \mathrm{C}-$ $K \alpha, \mathrm{N}-K \alpha$, and $\mathrm{O}-K \alpha$ peaks were clearly observed. Although the $\mathrm{N}-K \alpha$ peak cannot be observed in case of the SDD, it was possible to clearly observe the N-K $\alpha$ peak with concentration of less than $0.1 \%$ by using the STJ because the peak-to-background $(\mathrm{P} / \mathrm{B})$ ratio of the STJ is more than $10^{3}$. A full width at

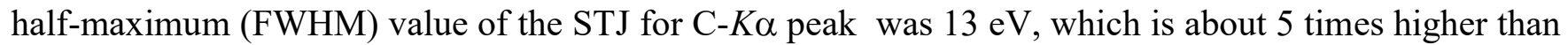
that of the SDD $(60 \mathrm{eV})$. Since the energy resolution of the STJ at $400 \mathrm{eV}$ is about $7 \mathrm{eV}$ [4], it was found that the peak broadening in the $\mathrm{C}-K \alpha$ peak from the sample occurred due to existing different chemical bonding states of carbon in there. This result indicates that it is possible to perform the chemical bonding state analysis by X-ray spectroscopy analyzer equipping the STJ.

\section{References:}

[1] R Wuhrer and K Moran, IOP Conf. Ser.: Mater. Sci. Eng. 109 (2016), p. 012019.

[2] DM Schlosser et al., Nucl. Inst. Methods in Phys. 624 (2010), p. 270.

[3] M.Ukibe et al., J. Low. Temp. Phys. 184 (2016), p. 200.

[4] G Fujii et al., X-ray spectrometry 46 (2017), p. 325.

[5]This work was supported by Innovative Science and Technology Initiative for Security, ATLA, Japan.

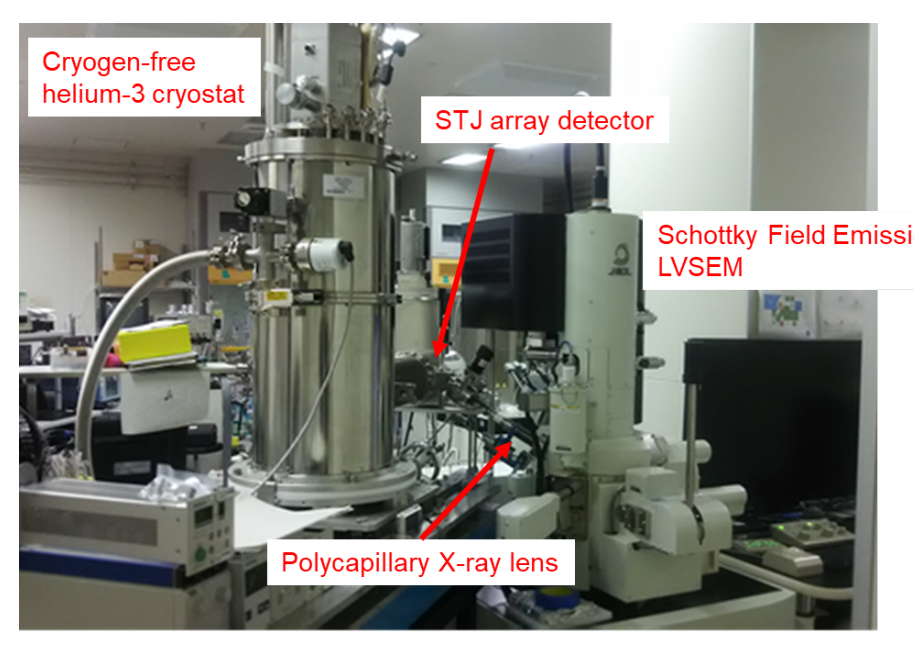

Figure 1. Picture of the SC-LVSEM.

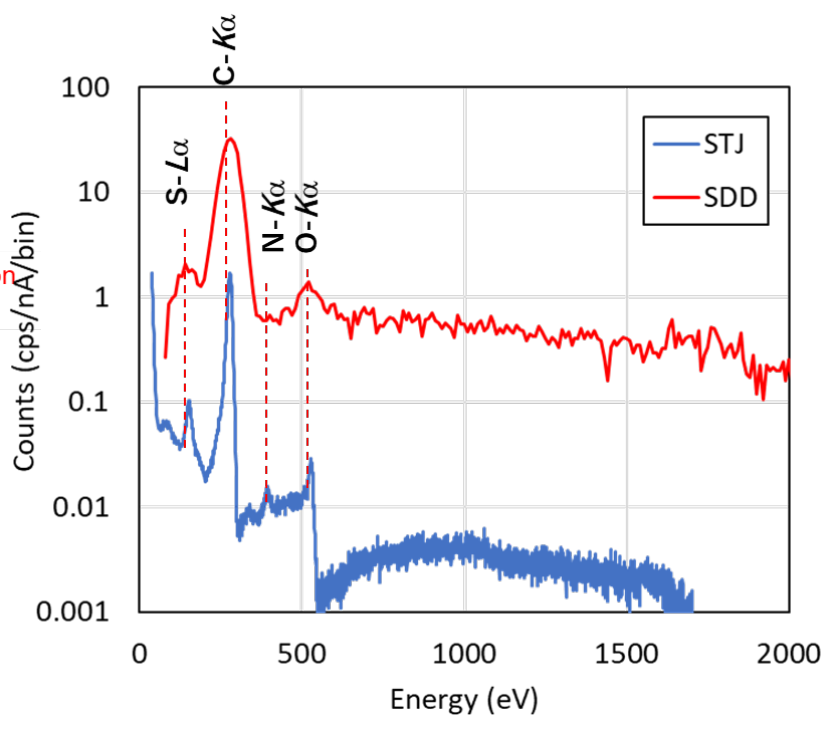

Figure 2. Energy spectra acquired using the STJ (Blue) and SDD (Red). 